\title{
Anabases
}

ANABASES Traditions et réceptions de l'Antiquité

17 | 2013

Varia

\section{L'Antiquité comme miroir inverse des « misères de ce temps » : usages des références antiques dans la pensée politique du XVI ${ }^{\mathrm{e}}$ siècle en France}

\section{Bérengère Basset}

\section{(2) OpenEdition}

\section{Journals}

Édition électronique

URL : http://journals.openedition.org/anabases/4170

DOI : $10.4000 /$ anabases. 4170

ISSN : 2256-9421

Éditeur

E.R.A.S.M.E.

Édition imprimée

Date de publication : 1 mars 2013

Pagination : 135-148

ISSN : 1774-4296

\section{Référence électronique}

Bérengère Basset, "L'Antiquité comme miroir inverse des « misères de ce temps » : usages des

références antiques dans la pensée politique du xvi ${ }^{e}$ siècle en France », Anabases [En ligne], 17 | 2013, mis en ligne le 01 avril 2016, consulté le 20 octobre 2019. URL : http://journals.openedition.org/ anabases/4170; DOI : 10.4000/anabases.4170 
Anabases 17 (2013), p. 135-148.

\section{L'Antiquité comme miroir inverse des " misères de ce temps": usages des références antiques dans la pensée politique du XVI ${ }^{\mathrm{e}}$ siècle en France}

BÉRENGÈRE BASSET

\section{Retour sur un titre}

EN GUISE D'introduction, nous prendrons un premier exemple, le passage des Essais de Montaigne qui nous a inspiré le titre de cet article. Il s'agit d'un extrait du chapitre "Des coches » qui traite de la conquête du Nouveau Monde. Avec les guerres de religion que connaît la France, c'est une des " misères de ce temps " par la cruauté et les exactions auxquelles cette conquête donne lieu. Montaigne vient de faire la peinture de ce "monde enfant ", sorte de nouvel âge d'or, pour en noter la " disparité " avec notre monde et expliquer ainsi la victoire remportée sur eux. Il poursuit en introduisant un troisième élément au sein de la relation binaire qu'il a posée :

"Que n'est tombée sous Alexandre, ou sous ces anciens Grecs et Romains, une si noble conquête, et une si grande mutation et altération de tant d'empires et de peuples, sous des mains, qui eussent doucement poli et défriché, ce qu'il y avait de sauvage, et eussent conforté et promu les bonnes semences, que nature y avait produit : mêlant non seulement à la culture des terres, et ornement des villes, les arts de deçà, en tant qu'elles y eussent été nécessaires, mais aussi, mêlant les vertus Grecques et Romaines, aux originelles du pays. Quelle réparation eût-ce été, et quel amendement à toute cette machine, que les premiers exemples et déportements nôtres, qui se sont présentés par-delà, eussent appelé ces peuples, à l'admiration, et imitation de la vertu, et eussent dressé entre eux et nous 
une fraternelle société et intelligence ? Combien il eût été aisé, de faire profit d'âmes si neuves, si affamées d'apprentissage, ayant pour la plus part de si beaux commencements naturels? Au rebours, nous nous sommes servis de leur ignorance, et inexpérience, à les plier plus facilement vers la trahison, luxure, avarice, et vers toute sorte d'inhumanité et de cruauté, à l'exemple et patron de nos meurs. Qui mit jamais à tel pris le service de la mercadence et de la trafique ? Tant de villes rasées, tant de nations exterminées, tant de millions de peuples, passés au fil de l'épée, et la plus riche et plus belle partie du monde bouleversée pour la négociation des perles et du poivre : mécaniques victoires. Jamais l'ambition, jamais les inimitiés publiques, ne poussèrent les hommes contre les uns les autres à si horribles hostilités, et calamités si misérables ${ }^{1}$. "

Au couple antagoniste formé par les habitants du Nouveau Monde et les Européens s'en substitue un autre qui oppose les conquérants de l'Antiquité à ceux du présent de Montaigne. De part et d'autre du " au rebours ", se font front, comme dans un miroir, deux images, et deux images qui s'inversent : d'un côté les modèles antiques, modèle "vertueux " de conquête que Montaigne s'enchante à évoquer mais qui est donné comme un irréel du passé ; de l'autre côté, le reflet dégradé, une conquête néfaste que blâme Montaigne et qui est, elle, de l'ordre du réel. D’un côté le rêve qui suscite la nostalgie de ces temps qui ne sont plus; de l'autre la déploration de ces temps qui sont. Montaigne opère un retour sur le passé et l'idéalise ${ }^{2}$ pour mieux accuser le présent. L'Antiquité se constitue comme une sorte d'utopie. Comme les utopies, elle est façonnée sur le mode du mundus inversus : le "polissage " de ce monde enfant s'inverse en son extermination, la vertu en la cupidité, la "fraternelle société " en "hostilités ". Mais là s'arrête le parallèle entre utopie et Antiquité. Cette dernière figure plutôt le miroir, comme "expérience mixte, mitoyenne ", située entre utopie et hétérotopie ${ }^{3}$, ainsi que l'analyse Michel Foucault :

« [...] et je crois qu'entre les utopies et ces emplacements absolument autres, ces hétérotopies, il y aurait sans doute une sorte d'expérience mixte, mitoyenne, qui serait le miroir. Le miroir, après tout, c'est une utopie, puisque c'est un lieu sans lieu. Dans le miroir, je me vois là où je ne suis pas, dans un espace irréel qui s'ouvre virtuellement derrière la surface, je suis là-bas, là où je ne suis pas, une sorte d'ombre qui me donne à moi-même

1 Montaigne, Essais, III, 6, Paris, Gallimard, coll. "Folio », 2009, p. 184-185. Nous utilisons cette édition qui modernise l'orthographe et la ponctuation afin de rendre le texte plus accessible.

2 Cette conquête vertueuse prêtée aux modèles antiques, et notablement à Alexandre, fait fi de certaines réalités, dont Montaigne avait cependant connaissance. Voir, pour Alexandre, le chapitre II, 36 des Essais. Bien qu'il fasse l'éloge du personnage, Montaigne note la cruauté dont il s'est montré capable envers les ennemis conquis (ibid., p. 754b).

3 Concept forgé par M. Foucault, l'hétérotopie désigne une localisation physique de l'utopie. Ce sont des espaces concrets qui hébergent l'imaginaire d'une société, ils en constituent comme le négatif. 
ma propre visibilité, qui me permet de me regarder là où je suis absent - utopie du miroir. Mais c'est également une hétérotopie, dans la mesure où le miroir existe réellement, et où il a, sur la place que j'occupe, une sorte d'effet en retour ${ }^{4}$. "

Toute idéalisée qu'elle est, l'Antiquité a existé réellement. Elle constitue ce que nous pourrions appeler une hétérotopie anachronique. Elle permet, dans le passage qui nous occupe, de prendre des distances avec le mythe de l'Âge d'or selon lequel est pensé le Nouveau Monde. Au temps statique de l'Âge d'or, qui est aussi celui de l'utopie, l'idéal que représente l'Antiquité substitue l'évolution : filant la métaphore du «monde enfant ", Montaigne conçoit l'action bénéfique des conquérants antiques comme une éducation ${ }^{5}$. Entre le monde imparfait, au sens premier du terme, que sont les terres nouvellement découvertes, et le monde dégradé qu'est l'Europe du Xvi siècle, l'Antiquité figure la perfection, comme état et comme processus. C'est un passé qui vient féconder le présent. Montaigne investit un motif essentiel du rapport qu'entretiennent les hommes de la Renaissance avec le passé gréco-romain. Conçue comme un modèle parfait, l'Antiquité est non seulement un miroir inverse, mais encore un point de mire. Il faut cependant ajouter que le passage que nous étudions montre le présent désarrimé de ce passé idéalisé. Comme une utopie, l'Antiquité rêvée par Montaigne est coupée du réel. Mais alors que l'utopie prétend agir sur le réel pour le réformer, il n'en va pas de même ici : l'irréel du passé semble condamner le présent à ne jamais retrouver les traces de l'idéal formulé. Le miroir inverse est un miroir brisé : si le Nouveau Monde figure, pour l'Europe du XVI ${ }^{\mathrm{e}}$ siècle, ce qui est radicalement autre, l'Antiquité est l'autre que nous aurions pu être, voire que nous aurions dû être. Cet extrait des Essais pose ainsi l'ambivalence de la relation à l'Antiquité qui se construit à la Renaissance, relation qui est à la fois d'identification, d'imitation et d'opposition. Il s'agira d'envisager ces différentes modalités de se rapporter au passé, d'étudier la dialectique qui se crée entre écart et proximité avec l'Antiquité, entre imitation et nostalgie, d'essayer de voir surtout comment les humanistes pensent l'Antiquité pour féconder leur présent. Les modalités qu'ils imaginent et envisagent nous semblent tributaires des configurations politiques. De notre parcours, Montaigne constitue le terminus ad quem. Il nous semblait intéressant d'ouvrir notre réflexion par lui car il utilise et transforme des motifs autour desquels s'est construite la relation des humanistes à l'Antiquité et les fait évoluer. C'est un peu l'archéologie de ces motifs que, à une échelle très réduite, nous élaborerons.

$4 \quad$ M. Foucault, Dits et écrits, II, Paris, 2001, p. 1575.

5 Voir les termes indiquant un processus : mutation, altération, réparation, amendement. 


\section{Au commencement était le miroir}

Les humanistes utilisent volontiers l'image du miroir pour traiter des relations qu'ils entretiennent avec l'Antiquité. C'est qu'ils sont tributaires, dans leur lecture du passé, d'une conception cicéronienne de l'histoire, définie comme magistra vitae. Il faut en effet revenir aux sens que revêt le terme "miroir " dans la langue du XVI siècle : à celui d' "image ", s'ajoutent ceux de "leçon, enseignement ", de " modèle " et enfin de " $\operatorname{type}^{6}$ ". C'est parce qu'elle délivre des leçons pour l'action et la conduite de la vie, qu'elle fournit des modèles, que l'histoire est conçue comme un miroir : importent moins la réalité et la vérité des exemples que leur capacité à incarner des types idéaux.

L'idéal que constitue l'Antiquité n'est pas forgé sur le mode de l'antithèse avec les " misères de ce temps ", il est une cible à atteindre. Le miroir devient le lieu d'une transformation de soi. C'est ce que nous voudrions montrer à travers l'Institution du prince, de Guillaume Budé. L'ouvrage, écrit en 1519 à destination de François I ${ }^{\text {er }}$ dont Budé recherche les faveurs et auprès de qui il plaide pour le développement de la culture, s'appuie sur les « dits » de Salomon, mais aussi et surtout sur les recueils d'apophtegmes de Plutarque. Il se présente explicitement comme un miroir du prince :

"Par lesquelles histoires on voit quasi comme un miroir les choses passées comme les présentes, par la considération desquelles, les hommes peuvent grandement acquérir prudence et mieux consulter les matières qui s'adonnent et offrent es [aux] conseils des princes, ainsi que nous voyons advenir tous les jours. Car il n'est rien qui tant fasse les hommes sages que d'entendre l'état du monde et la condition de nature humaine, et prévoir les cas qui peuvent échoir, et comment on y peut pourvoir et obvier, et est ce [c'est ce] que les historiens enseignent ${ }^{7}$."

Ce qui nous intéresse davantage c'est la manière dont Budé "façonne » la matière antique qu'il emprunte pour la placer au service de la parénèse royale. Constitués en modèles, les exemples de l'Antiquité sont aussi modelés. Il nous semble que l'on peut identifier, dans l'Institution du prince, trois types de "déformations " : l'adaptation aux réalités présentes, l'interprétation au service d'une thèse, enfin l'invention d'un événement ou d'un dit. De la première à la troisième de ces modalités déformantes, l'écart avec la réalité historique se creuse, mais plus il se creuse plus les exemples de l'Antiquité sont féconds pour le présent.

6 Voir l'article que consacre à ce terme le Dictionnaire de la langue du XVI ${ }^{e}$ siècle de Huguet.

7 Budé, Institution du prince, ms Arsenal, d'après la transcription de C. BonTEMS, in C. Bontems, L.-P. Raybaud et J.-P. Brancourt, Le prince dans la France des XVI et $X V I I^{e}$ siècles, Paris, 1965, f. 25r-25v. Nous modernisons l'orthographe et la ponctuation pour rendre le texte plus lisible, et ajoutons, entre crochets, quelques éclaircissements. 
La première modalité, l'adaptation, porte sur les realia antiques qui sont accommodés au présent. La déformation est essentiellement de nature culturelle, mais peut se charger d'une dimension idéologique. Budé utilise ainsi un épisode de l'histoire antique mettant en scène Philippe de Macédoine, présenté de la manière suivante dans sa source, les Apophtegmes des rois et des généraux de Plutarque :

"Lorsque Harpalos proposa, en faveur de son parent et ami Kratès, poursuivi pour méfaits, que celui-ci payât spontanément l'amende, mais que l'arrêt lui fût épargné afin qu'il échappât aux sarcasmes, "Mieux vaut, répondit Philippe, qu’il soit lui-même décrié plutôt que moi à cause de lui ${ }^{8 ”}$."

Dans l'Institution du prince, l'anecdote s'inscrit dans les procédures en vigueur au XVI ${ }^{\mathrm{e}}$ siècle, notamment le "Grand Conseil » qui assiste le roi et dont les membres se dotent, par une ordonnance remontant à Charles VII, d'une autorité judiciaire qui en fait un corps indépendant du roi et des parlements. Ce corps judiciaire se voit de plus en plus chargé des causes dites évoquées, " c'est-à-dire les procès qu'ils arrachent (évoquent du latin evocare, appeler à soi, faire venir) aux tribunaux royaux qui devraient normalement les connaître, mais dont les juges pourraient être trop indépendants ou partiaux ${ }^{9}$ ». C'est précisément cette pratique et les abus auxquels elle donne lieu que Budé dénonce par les modifications qu'il opère sur le texte de Plutarque :

"Entre ses gentils hommes de chambre ou autres ses mignons, était un nommé Harpalus. Ce gentil homme avait un prochain [proche] parent qui était attrait [cité] en justice et appréhendé pour un cas de violence qu'il avait fait. Harpalus averti qu'il était en voie de condamnation, fit requête au roi que son plaisir fût de évoquer la cause devant lui en la suite de sa cour ; tendant à fin [cherchant à] d'assoupir la matière, et que les parties poursuivantes par ce moyen se fâchassent et attendissent du procès et des tergiversations de leur partie, et abandonnassent leur poursuite plutôt que de suivre la cour du roi, où ils n'eussent pu facilement trouver conseil et faveur pour obtenir sentence selon le droit qu'ils prétendaient avoir, en disant au roi par ledit gentil homme : "Si mon cousin est condamné, il est diffamé à jamais et perdra réputation entre les hommes de nom et d'honneur." Alors le roi lui répondit ces paroles ou autres semblables : "Quel remède si vaut-il mieux : que ton cousin soit diffamé par son outrage et méfait, [ou] que je fasse mal parler de moi pour avoir fait ceste injustice en faveur de lui et de toi ${ }^{10}$ ?” "

Les realia du XVI $\mathrm{e}^{\mathrm{e}}$ siècle s'introduisent au sein de l'exemple antique, passé et présent se superposent : le premier permet de tenir un discours sur le second. L'adaptation revêt une portée idéologique que souligne Claude Bontems dans un bref commentaire de ce passage : "De même [Budé] se fait l'écho des plaintes fréquentes visant la pratique

8 Plutarque, Euvres morales, Paris, 2003 [2 $2^{\mathrm{e}}$ tirage], trad. F. Funrmann, 179a.

9 A. Jounnna, La France du XVI siècle 1483-1598, Paris, 2006, p. 142.

10 Budé, f. 49v-50r. Nous soulignons. 
abusive des évocations devant le conseil privé, qui n'étaient qu'un moyen dilatoire ${ }^{11}$. " L'adaptation du texte de Plutarque mue Budé en conseiller du prince.

Cette fonction, il la remplit plus encore quand il intervient sur les exemples antiques pour en donner une interprétation personnelle. La déformation passe par une amplification qui « déplie » l'exemple et l'oriente. Ainsi de l'utilisation d'un dit d'Antigonus ainsi formulé dans la source antique, Plutarque à nouveau :

"Se retirant un jour devant l'avance des ennemis, il déclara qu'il ne fuyait pas mais qu'il poursuivait au contraire son intérêt qui se trouvait derrière ${ }^{12}$.»

Budé le développe ainsi :

"Une autre fois en une bataille par terre, il sentit que la fortune tournait sur lui, et pour ce [pour cette raison] il retira ses gens sagement sans soi étonner [s'étonner], et puis petit à petit reculait en fuyant ses ennemis. Et pour ce qu'en faisant [comme en faisant cela] il aperçut aucuns des plus courageux capitaines s'émerveillaient [s'étonnaient] et contristoient [s'attristaient] pour ce qu'il fuyait, craignant la honte de la fuite, alors : "Non, non, dit-il, ne vous donnez trop grand merveille [ne vous étonnez pas outre mesure] de mon fait, car afin que vous l'entendez, je ne fuis pas devant mes ennemis, mais je veux bien suivre mon avantage si je puis, lequel je vois et entends [que je vois] se retirer de ceste part." Et par cette parole honnêtement donnait à entendre que aucunes fois [parfois] un vaillant chef de guerre pour sauver ses gens, doit reculer en tenant bon ordre, quand il voit clairement que la demeure [la station] et attente est dommageable ${ }^{13}$."

Dans la version laconique de Plutarque, il est difficile de déterminer si le personnage illustre une vertu ou un vice. Budé ôte toute ambiguïté : l'attitude du roi devient exemplaire par la justification qu'il en donne dans sa réplique et par le commentaire élogieux dont l'accompagne Budé. Antigonus, loin d'être lâche, se montre capable de saisir le kairos, et Budé, à travers lui, récuse l'héroïsme guerrier. Il " tord " le dit au service des thèses qu'il cherche à promouvoir.

L'instrumentalisation des exemples antiques conduit Budé à franchir les frontières de la déformation par interprétation et à inventer le matériau dont il a besoin. Relatant un épisode de la geste d'Alexandre, la proposition que lui fait Darius d'un partage du pouvoir, l'Institution du prince présente ainsi la réaction du Macédonien :

"Alexandre, roi de souveraine et insupérable [incomparable] magnanimité, quand il eut ouï cette ambassade, assembla ses grands barons et principaux conseillers, et mit ceste matière en conseil, non pas pour en délibérer, car il savait bien ce qu'il avait à faire, mais pour ouïr les opinions de son conseil, et entendre le cœur de ses gens, et aussi

11 C. Bontems, "L'institution du prince de Guillaume Budé », in Le prince dans la France des XVI et $X V I I^{e}$ siècles, p. 69.

$12183 \mathrm{~d}$.

13 f. 58 r. 
pour montrer le sien. Quand il eut ouï parler les autres, il vint à demander l'opinion de Parménion son lieutenant général et conducteur de l'avant-garde, et en qui il avait toute fiance, [...]. Après donc que le roi eut commandé audit lieutenant de dire ce qu'il lui en semblait, il parla honnêtement en guerroyeur [en homme de guerre] et sagement en déduisant les raisons de redoubler l'événement de ceste entreprise, les hasards qui peuvent survenir en guerre, et la variété de fortune, et finalement, en concluant que le roi ne pouvait plus avant souhaiter, à son avis, que ce qu'on lui offrait. [Il] dit : "Sire, si j'étais le roi Alexandre, je prendrais ce parti et accepterais l'offre qu'on vous fait." Alors Alexandre qui ne faisait estime d'autre chose que de gloire laquelle il s'efforçait d'acquérir par tous moyens, va répondre : "Foi de roi, dit-il, si j'étais Parménion je ferais ce que vous avez dit, mais pour ce que je suis Alexandre conquéreur de renommée et avaricieux [avide] de gloire, assuré de pauvreté et contempteur d'[méprisant l'] or et d'argent, je ne le ferai nullement.” "

La convocation d'un conseil par Alexandre - relatée avec un lexique qui ancre l'épisode dans la France du XVI ${ }^{\mathrm{e}}$ siècle - et l'argumentation très nuancée et très sensée de Parménion sont pures inventions. Ni l'une ni l'autre ne sont mentionnées par les sources historiques, Quinte Curce ou Plutarque. À une époque où s'opère le passage d'une monarchie consultative à une monarchie absolue, l'ajout de Budé n'est pas dénué de dimension idéologique. Rapporté aux réalités politiques du XVI siècle, l'exemple voit son sens infléchi : il montre que la consultation du conseil garantit des décisions raisonnables, permet de se prémunir contre l'arbitraire.

Érudition et recréation à des fins idéologiques se mêlent ainsi. C'est que l'Antiquité doit révéler et féconder les vertus innées du roi. Budé conclut sur ces considérations :

"Si mon effort et labeur vous vient à gré, il ne se peut faire qu'il n'excite, en votre très noble courage plein de générosité des mœurs royales et quasi héroïque, une très honnête et fervente émulation des choses louables et recommandables qui sont récitées dedans, en augmentant et stimulant et mettant en exercice les vertus dont dieu et nature ont mis la semence et native inclination en vous par grande largesse et bénignité ${ }^{14}$. "

On retiendra l'image des semences déjà présente dans le passage de Montaigne que nous avons cité : la lecture de l'Antiquité réalise l'action bénéfique qu'auraient, selon Montaigne, produite les Anciens s'il leur avait été donné d'accomplir la conquête du Nouveau Monde. La restitution de l'Antiquité complète l'œuvre de la nature : elle fait germer les graines que celle-ci a semées. 


\section{Restituer l'Antiquité : un processus de "germination "}

Cette image des semences pour désigner les bonnes qualités que la nature a placées en nous, nous la retrouvons dans le Discours de la servitude volontaire de La Boétie :

"Pour cette heure je ne penserai point faillir en disant cela, qu'il y a en notre âme quelque naturelle semence de raison, laquelle, entretenue par bon conseil et coutume, florit en vertu, et, au contraire, souvent ne pouvant durer contre les vices survenus, étouffée, s'avorte ${ }^{15}$."

Le topos remonte aux Tusculanes de Cicéron dans une assimilation entre la culture d'un champ et l'action bénéfique de la philosophie sur notre âme ${ }^{16}$. Les humanistes réinvestissent la comparaison cicéronienne en faisant jouer à la lecture des exemples de l'Antiquité le rôle dévolu à la philosophie. C'est la fonction à laquelle elle nous semble également vouée dans le Discours de la servitude volontaire. L'ouvrage de La Boétie n'est cependant pas produit dans le même contexte politique que celui de Budé et n'entretient pas les mêmes relations au pouvoir, il en résulte une inflexion dans le rôle confié à l'Antiquité ${ }^{17}$. Si l'on ne saurait affirmer que Le Discours vise à dénoncer le pouvoir en place, force est de constater qu'il est une charge contre la tyrannie et un appel à la liberté. Surtout, La Boétie s'interroge sur ce qui a pu faire perdre aux hommes leur appétence pour la liberté et les faire sombrer dans ce "monstre de vice ${ }^{18}$ ", question à laquelle il ne trouve pas de réponse, sinon celle qui consiste à avancer quelque « malencontre $^{19}$ ". La restitution de l'Antiquité nous semble servir une forme de réparation de cette malédiction. Car l'exception que constitue l'Antiquité, du moins une partie de l'Antiquité, dans cet invariant commun à toutes les sociétés qu'est la servitude volontaire, cette exception donc, pour n'être pas nécessairement réelle, ne saurait être pure rhétorique. La lutte d'Athènes et Sparte contre l'ennemi perse offre à La Boétie des preuves du désir de la liberté qui nous anime dans ce que l'on pourrait appeler un « état

15 La Boétie, Le Discours de la servitude volontaire, Paris, GF Flammarion, 1983, p. 140.

16 Voir Cicéron, Tusculanes, livre II, 13 : Atque, ut in eodem simili verser, ut ager quamvis fertilis sine cultura fructuosus esse non potest, sic sine doctrina animus; ita est utraque res sine altera debilis. Cultura autem animi philosophia est; haec extrahit vitia radicitus et praeparat animos ad satus accipiendos eaque mandat iis et, ut ita dicam, serit, quae adulta fructus uberrimos ferant.

17 Sur les sources antiques utilisées et reprises par La Boétie, voir : L. Delaruelle, "L'inspiration antique dans le Discours de la servitude volontaire", Revue d'histoire littéraire de la France, 1910, p. 34-72 ; O. Guerrier, "Aux origines du Discours de la servitude volontaire: autour d'un mot de Plutarque ", in O. GUERrIER (dir.), Moralia et Euvres morales à la Renaissance, Paris, 2008, p. 237-251.

18 La Boétie, p. 135.

19 "Quel malencontre a été cela, qui a pu tant dénaturer l'homme, seul né, de vrai, pour vivre franchement, et lui faire perdre la souvenance de son premier être, et le désir de le reprendre ", p. 143. 
de nature ", mais c'est à un exemple de l'Antiquité romaine, celui de Caton d'Utique, que nous voudrions nous intéresser. La Boétie le puise dans la Vie de Caton:

"Caton l'Uticain, étant encore enfant et sous la verge, allait et venait souvent chez Sylla le dictateur, tant pour ce qu'à raison du lieu et maison dont il était, on ne lui refusait jamais la porte, qu'aussi ils étaient proches parents. Il avait toujours son maitre quand il y allait, comme ont accoutumé les enfants de bonne maison. Il s'aperçut que, dans l'hôtel de Sylla, en sa présence ou par son consentement, on emprisonnait les uns, on condamnait les autres ; l'un était banni, l'autre étranglé ; l'un demandait la confiscation d'un citoyen, l'autre la tête; en somme, tout y allait non comme chez un officier de ville, mais comme chez un tyran de peuple; et c'était non pas un parquet de justice, mais un ouvroir de tyrannie. Si dit lors à son maître ce jeune gars : "Que ne me donnez-vous un poignard ? Je le cacherai sous ma robe ; j'entre souvent dans la chambre de Sylla avant qu'il soit levé, j'ai le bras assez fort pour en dépêcher la ville." Voilà certes une parole vraiment appartenant à Caton ; c'était un commencement de ce personnage, digne de sa mort ${ }^{20}$. "

Le personnage n'est nullement héroïsé, il n'est pas constitué en modèle à suivre appelant à la révolte et au tyrannicide. On le mesure en lisant ce qui suit :

Et néanmoins qu'on ne die ni son nom ni son pays, qu'on conte seulement le fait tel qu'il est, la chose même parlera et jugera l'on, à belle aventure, qu'il était Romain, et né dedans Rome, et lors qu'elle était libre ${ }^{21}$.

Le locuteur, Caton, s'efface devant la parole qu'il a prononcée ; elle est le signe moins de l'excellence de son âme que d'une société libre, qui a existé hors du " malencontre $^{22} »$. La Boétie précise la portée qu'il donne à cet exemple :

"A quel propos tout ceci ? Non pas certes que j'estime que le pays ni le terroir y fassent rien ; car en toutes contrées, en tout air, est amère la sujétion, et plaisant d'être libre ; mais parce que je suis d'avis qu'on ait pitié de ceux qui, en naissant, se sont trouvés le joug sous le col, ou bien que si on les excuse, ou bien que leur on pardonne, si n'ayant vu seulement l'ombre de la liberté et n'en étant point avertis, ils ne s'aperçoivent pas point du mal que ce leur est d'être esclaves ${ }^{23}$."

Et il initie un développement sur la coutume venue ensevelir notre nature, "première raison de la servitude volontaire ${ }^{24}$ ". L'exemple de Caton a ainsi pour

20 La Boétie, Le Discours de la servitude volontaire, p. 149.

21 Ibid.

22 Cette société d'avant la malencontre n'est cependant pas celle de l'époque des faits relatés. L'exemple de Caton constitue une sorte de moment charnière, le moment de basculement - que La Boétie se garde bien de dater et qui n'est peut-être qu'un mirage - de la société libre dans celle de la tyrannie et de la servitude volontaire.

23 La Boétie, Le Discours de la servitude volontaire, p. 149.

24 La Boétie, Le Discours de la servitude volontaire, p. 149-150. 
fonction de faire germer à nouveau la semence de liberté que la nature a placée en nous, voire d'introduire une nouvelle semence si celle de la nature a dégénéré. Dans une société où la servitude volontaire est devenue la norme, la "pratique " de l'Antiquité devient le seul moyen non seulement d'entretenir le souvenir de ce qu'on a perdu, mais encore de connaître ce bien perdu. Le rappel de l'amour des Anciens pour la liberté procure à La Boétie le plaisir que lui donnerait le souvenir de la liberté s'il l'avait connue. Il note en effet :

"On ne plaint jamais ce que l'on n'a jamais eu, et le regret ne vient point sinon qu'après le plaisir, et toujours est avec la connaissance du mal, la souvenance de la joie passée ${ }^{25}$."

Ce plaisir, cette joie, qu'il n'a pas connus et sur lesquels repose cependant l'attachement à la liberté, c'est l'Antiquité qui va les lui donner. L'exemple des Spartiates envoyés en ambassade au Perse Indarne, exemple qui précède immédiatement celui de Caton, est introduit par une formule riche de sens quand on la replace dans le cadre de la réflexion menée : "Je prends plaisir de ramentevoir ${ }^{26}$. "C'est un écho à cette "souvenance de la joie passée " qui nous fait connaître le mal que l'on vit dans le présent. L'Antiquité est certes conçue comme un miroir inverse des "misères de ce temps » que sont, pour La Boétie, la tyrannie et la servitude volontaire. Mais il s'agit moins d'accuser le présent que de retrouver une image de la nature de l'homme qui s'est perdue. Le reflet qu'offre le miroir de l'Antiquité fait alors l'objet d'une quête, voire d'une reconquête. Si, comme l'affirme Laurent Gerbier, la liberté est " un pur processus, un état de désir permanent ${ }^{27}$ ", la lecture de l'Antiquité entre pleinement dans ce processus en entretenant cet état de désir, lui permettant de se faire permanent. L'Antiquité autorise le déploiement d'un espace mental où va s'exercer la liberté quand les conditions politiques et sociales de sa réalisation n'existent plus ${ }^{28}$. S'il y a " créativité imaginative " de La Boétie dans sa restitution de l'Antiquité, cela relève de la "liberté imaginative " qu'elle permet, d'une culture en soi des semences de liberté que nature a placées en nous. Et il faut encore ajouter que les exemples des anciens donnent une existence historique sinon à l'état de liberté du moins à l'expression du désir de liberté ; elle fait échapper ce refus de la servitude à l'utopie ou à la pure abstraction.

25 La Boétie, Le Discours de la servitude volontaire, p. 150.

26 La Boétie, Le Discours de la servitude volontaire, p. 147.

27 L. Gerbier, "Les paradoxes de la nature dans le Discours de la servitude volontaire de La Boétie ", in Discours de la servitude volontaire, éd. A. et L. TOURNON suivi de Les paradoxes de la servitude volontaire, Paris, 2002, p. 128.

28 Voir les développements sur les "quelques-uns mieux nés ": "Ceux-là, quand la liberté serait entièrement perdue et toute hors du monde, l'imaginent et la sentent en leur esprit, et encore la savourent " (La Boétie, p. 151). 


\section{Retour sur Montaigne : s'en tenir " aux plus humaines imitations"}

Nous voudrions terminer ce bref parcours en opérant un retour sur Montaigne. Si nous sommes partis de lui pour traquer en amont les images que nous l'avions vu investir, il s'agit à présent, sur de très brefs exemples, de voir comment il prolonge et revisite le rapport aux anciens initié par ses devanciers immédiats. Car si notre exemple inaugural nous a conduit à montrer dans les Essais un passé désarrimé du présent, nous voudrions nuancer cette image en mettant au jour l'" action » du passé sur le présent de Montaigne.

On retrouve, chez lui, l'image des semences pour penser le rapport aux Anciens. Ainsi au chapitre 32 du livre II :

"Moi je considère aucuns hommes fort loin au-dessus de moi : nommément entre les anciens : et encore que je reconnaisse clairement mon impuissance à les suivre de mes pas, je ne laisse pas de les suivre à vue, et juger les ressorts qui les haussent ainsi : desquels je aperçois aucunement en moi les semences : comme je fais aussi de l'extrême bassesse des esprits, qui ne m'étonne, et que je ne mécrois non plus. Je vois bien le tour que celles là se donnent pour se monter, et admire leur grandeur : et ces élancements que je trouve très beaux, je les embrasse ; et si mes forces n'y vont, au moins mon jugement s'y applique très volontiers ${ }^{29}$."

Le passage montre que l'image n'a plus le sens qu'elle avait chez les prédécesseurs. Montaigne n'établit plus un rapport mimétique avec les modèles du passé. Ils ne sont pas pour lui des miroirs. Ils se prêtent à un exercice de jugement qui permet une prise de conscience de nos limites. Ils nous invitent à nous mesurer à notre aune, à accepter notre condition. Cette perspective se déploie pleinement dans la relation établie avec la figure de Caton d'Utique :

"Quant à moi, je puis désirer en général être autre : je puis condamner et me déplaire de ma forme universelle : et supplier Dieu pour mon entière réformation et pour l'excuse de ma faiblesse naturelle. Mais cela : je ne le dois nommer repentir ce me semble : non plus que le déplaisir de n'être, ni Ange ni Caton. Mes actions sont réglées, et conformes à ce que je suis, et à ma condition. Je ne puis faire mieux. Et le repentir, ne touche pas proprement les choses qui ne sont pas en notre force, oui bien le regretter. J'imagine infinies natures plus hautes et plus réglées que la mienne ; je n'amende pourtant mes facultés ${ }^{30}$. "

29 Montaigne, Essais, II, 32, p. 570-571. Nous soulignons.

30 Montaigne, Essais, III, 2, p. 47. 
Les hommes illustres de l'Antiquité ne constituent pas un patron sur lequel se mouler, ils permettent de définir un patron intériorisé, conforme à ce que nous sommes, dans une adhésion lucide et raisonnée à soi :

"Nous autres principalement, qui vivons une vie privée, qui n’est en montre qu’à nous, devons avoir établi un patron au-dedans, auquel toucher nos actions : Et, selon icelui, nous caresser tantôt, tantôt nous châtier. J'ai mes lois et ma cour, pour juger de moi, et m'y adresse plus qu'ailleurs ${ }^{31}$."

C'est la définition du for intérieur que nous propose Montaigne. L'expression est en effet empruntée au domaine de la justice et trouve un équivalent dans la formule de l'« interne juridiction». Montaigne semble prolonger le mouvement d'intériorisation des modèles antiques initié par La Boétie.

Aussi participent-ils à la réflexion menée sur l'" équilibre " à établir entre les domaines privé et public, entre l'utile et l'honnête qui font la matière du chapitre III, 1. C'est ce dont témoigne l'utilisation, dans ce chapitre, de la figure d'Épaminondas. Ce dernier vient définir une ligne de conduite " propre au temps ", à savoir les guerres civiles, invitant à préférer l'honnête à l'utile :

"Ne craignons point, après un si grand précepteur, d'estimer qu'il y a quelque chose illicite contre les ennemis mêmes : que l'intérêt commun ne doit pas tout requérir de tous, contre l'intérêt privé $[\ldots]$ : Et que toutes choses, ne sont pas loisibles à un homme de bien, pour le service de son Roi, ni la cause générale et des lois. [...] C’est une instruction propre au temps : Nous n'avons que faire de durcir nos courages par ces lames de fer, c'est assez que nos épaules le soient : c'est assez de tremper nos plumes en encre, sans les tremper en sang. Si c'est grandeur de courage, et l'effet d'une vertu rare et singulière, de mépriser l'amitié, les obligations privées, sa parole, et la parenté, pour le bien commun, et obéissance du magistrat : C'est assez vraiment, pour nous en excuser, que c'est une grandeur qui ne peut loger en la grandeur du courage d'Epaminondas ${ }^{32}$."

On pourra être surpris, compte tenu de nos précédentes analyses, du magistère dont est doté Épaminondas. Son rôle consiste moins, cependant, à dicter une ligne de conduite qu'à cautionner une prise de position personnelle en lui donnant une assise. Le patron intérieur s'affirme dans l'exercice de jugement auquel donne lieu la figure du grand homme. Montaigne l'utilise pour donner une nouvelle définition du courage. À partir des témoignages légués sur le Thébain - principalement le Démon de Socrate de Plutarque -, Montaigne fabrique une figure oxymorique, mélange harmonieux de force et d'énergie au combat d'une part, de douceur et de bonté d'autre part. Épaminondas parvient à imposer à l'utile - les impératifs de la guerre - les droits et les devoirs de la conscience. Mais il reste une figure exceptionnelle : cette capacité à harmoniser l'utile

31 Montaigne, Essais, III, 2, p. 38-39.

32 Montaigne, Essais, III, 1, p. 33. 
et l'honnête, la fureur guerrière et la douceur extrême " n'appartient qu'à lui ". " Nous autres petits ", dirait Montaigne, si nous y parvenons, c'est " miracle " et ce n'est qu'une "image de justice " : la chose se réalise non par un effet de notre complexion, comme pour Épaminondas, mais par un hasard qui pourrait être le masque de quelque providence. C'est pourquoi Montaigne n'invite pas à se modeler sur Épaminondas, se contentant de s'appuyer sur sa caution pour refuser le primat de l'intérêt commun sur l'intérêt privé. La figure d'Épaminondas se voit alors opposer celle de César :

"J'abomine les enhortements enragés, de cette autre âme déréglée,

dum tela micant, non vos pietatis imago

Ulla, nec adversa conspecti fronte parentes

Commoveant; vultus glagio turbate verendos.

Ôtons aux méchants naturels, et sanguinaires, et traîtres, ce prétexte de raison : Laissons

là cette justice énorme, et hors de soi, et nous tenons aux plus humaines imitations ${ }^{33}$. "

Les "plus humaines imitations ", opposées à la monstruosité de César, font écho à " l'humanité » que Montaigne a perçue en Épaminondas et qu'il joignait à sa bonté ${ }^{34}$ : "S'en tenir aux plus humaines imitations ", consiste moins à suivre un modèle qu'à retrouver en lui l'homme que nous sommes, qu'à conquérir, à travers lui, notre dignité d'homme. La restitution de l'Antiquité aurait pour enjeu de faire de nous des hommes, tâche qui demande humilité et exigence de tous les instants.

Nous n'avons présenté que quelques modalités des utilisations auxquelles se prête l'Antiquité dans la réflexion politique des humanistes. Le travail demanderait à être nuancé et complété. L'Antiquité est sans doute un miroir, mais un miroir à multiples facettes. Si cette étude partielle autorise à tirer quelque conclusion, elle consisterait à dire que l'Antiquité est pour les humanistes un " objet " vivant. Elle n'est pas pure érudition, mais est aux prises avec le présent qu' elle vient façonner et enrichir, dans les domaines publics et privés selon les cas et les positionnements politiques.

Dans ses Considérations inactuelles, Nietzsche, réfléchissant à " l'utilité et [aux] inconvénients de l'histoire pour la vie ", plaide en faveur d'une histoire qui ne soit pas amas de connaissances mais création au service de la vie, assumant que le passé, dans ce projet, soit "déformé, enjolivé, et ainsi rapproché de la libre invention poétique ${ }^{35}$ ". Il légitime cette position, hétérodoxe et quelque peu provocatrice, par le souci d'établir ce qu'il appelle une "vraie culture ", une culture qui soit un véritable processus intérieur

33 Montaigne, Essais, III, 1, p. 33. La citation est empruntée à Lucain, Pharsale, VII, 320-322 : "Tant que les lances brilleront, n'éprouvez aucune ombre de pitié, que les visages de vos parents en face de vous ne vous émeuvent pas : frappez de votre glaive ces traits vénérés ".

34 "Il mariait aux plus rudes et violentes actions humaines, la bonté et l'humanité " (Montaigne, III, 1, p. 31).

35 Nietzsche, "De l'utilité et des inconvénients de l'histoire pour la vie ", Considérations inactuelles, Paris, 2007, trad. de P. Rusch, p. 106 
et non un simple "savoir dont on se gave ». L'image des semences, qui est apparue si matricielle dans le cadre de notre étude, tend à montrer que la restitution du passé que pratiquent les humanistes est au service de cette " vraie culture " :

"L'homme moderne finit par avoir l'estomac chargé d'une masse énorme de connaissances indigestes qui, comme il est dit dans le conte, se heurtent et s'entrechoquent dans son ventre. Ce bruit révèle la caractéristique la plus intime de cet homme moderne : la remarquable opposition - inconnue aux peuples anciens - entre une intériorité à laquelle ne correspond aucune extériorité et une extériorité à laquelle ne correspond aucune intériorité. Le savoir, dont on se gave sans, le plus souvent, en éprouver la faim, parfois même malgré un besoin contraire, n'agit plus comme une force transformatrice orientée vers le dehors, il reste dissimulé dans une certaine intériorité chaotique, que l'homme moderne désigne avec une singulière fierté comme sa "profondeur" spécifique. On dit alors qu'on possède le contenu, et qu'il ne manque plus que la forme; mais c'est là, pour tout être vivant, une opposition totalement inappropriée. Et c'est justement parce que notre culture moderne ne peut absolument pas être comprise sans cette opposition, qu'elle ne constitue pas une réalité vivante, mais seulement une sorte de savoir sur la culture. On s'en tient à l'idée de la culture, au sentiment de la culture, on ne s'engage pas dans une culture déterminée. En revanche, ce qu'on exprime réellement, ce qu'on manifeste au-dehors en actes visibles se réduit bien souvent à une froide convention, une pauvre imitation ou même une caricature grossière. [...] Le processus intérieur, voilà maintenant la vraie "culture" 36 ".

Bérengère BASSET

PLH-ELH

Université de Toulouse (UTM)

Pavillon de la Recherche

5, allées Antonio Machado

F-31058 Toulouse Cedex 9

basset.berengere@wanadoo.fr 